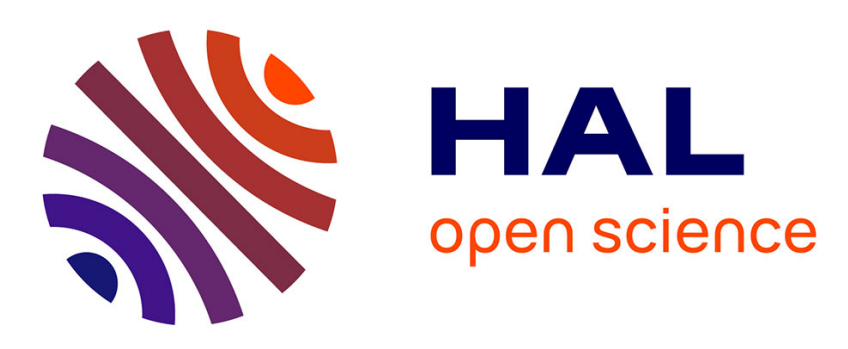

\title{
Safer and Performing Energetic Materials Based on Polyaniline-Doped Nanocomposites
}

Pierre Gibot, Arnaud Bach, Loïc Vidal, Fabien Schnell, Roger Gadiou, Denis

Spitzer

\section{- To cite this version:}

Pierre Gibot, Arnaud Bach, Loïc Vidal, Fabien Schnell, Roger Gadiou, et al.. Safer and Performing Energetic Materials Based on Polyaniline-Doped Nanocomposites. Journal of Energetic Materials, 2016, 35 (2), pp.136-147. 10.1080/07370652.2016.1210697 . hal-02349074

\section{HAL Id: hal-02349074 \\ https://hal.science/hal-02349074}

Submitted on 31 Jan 2022

HAL is a multi-disciplinary open access archive for the deposit and dissemination of scientific research documents, whether they are published or not. The documents may come from teaching and research institutions in France or abroad, or from public or private research centers.
L'archive ouverte pluridisciplinaire HAL, est destinée au dépôt et à la diffusion de documents scientifiques de niveau recherche, publiés ou non, émanant des établissements d'enseignement et de recherche français ou étrangers, des laboratoires publics ou privés. 


\title{
Safer and performing energetic materials based on polyaniline-doped nanocomposites
}

\author{
Pierre Gibot ${ }^{\mathrm{a}, *}$, Arnaud Bach ${ }^{\mathrm{a}, \mathrm{b}}$, Loic Vidal ${ }^{\mathrm{b}}$, Fabien Schnell ${ }^{\mathrm{a}}$, Roger Gadiou ${ }^{\mathrm{b}}$, \\ Denis Spitzer $^{\mathrm{a}}$ \\ a. Laboratory of Nanomaterials for Systems under Extreme Stress, \\ CNRS-ISL-UNISTRA UMR 3208, French-German Research Institute of Saint-Louis, \\ 5 rue du Général Cassagnou, BP70034, 68301 Saint-Louis, France \\ b. Institute of Materials Science of Mulhouse (IS2M), CNRS UMR 7361, \\ University of Haute Alsace (UHA), 15 rue J. Starcky, BP2488, 68057 Mulhouse, France
}

Short title: Polyaniline as desensitiser for nanothermites.

Type of article: Research paper.

*Corresponding author. Tel: +33 (0)3.89.69.58.77. E-mail: pierre.gibot@isl.eu (Pierre Gibot)

Co-authors:

arnaud.bach@isl.eu; Tel.: +33 (0)3.89.69.58.75

loic.vidal@uha.fr; Tel.: +33 (0)3.60.89.87.37

fabien.schnell@isl.eu; Tel.: +33 (0)3.89.69.51.70

roger.gadiou@uha.fr; Tel.: +33 (0)3.89.60.87.19

denis.spitzer@isl.eu; Tel.: +33 (0)3.89.69.50.75 


\begin{abstract}
Nanothermites, combining a fuel with an oxidizer at the nanoscale, represent a class of energetic material that has been attracting increasing attention over the past decade. This intensive interest is due to their tuneable pyrotechnic performance, making the materials promising candidates for ordnance applications. However, the extreme mechanical and electrostatic sensitivities of energetic composites make handling them hazardous. In this study, a realistic desensitisation method is suggested via the addition of polyaniline while maintaining an interesting combustion velocity in contrast to the literature values. This investigation claims a major scientific breakthrough in the preparation of safer energetic nanocomposites.
\end{abstract}

Keywords: Nanothermites; Polyaniline; Desensitisation; Energetic nanocomposites. 


\section{Introduction}

Since 2006, the European laws on chemicals have reinforced the Regulation on Registration, Evaluation, Authorization and Restriction of Chemicals (REACH) [1]. The aim of these laws is to improve the protection of human health and the environment by banning hazardous materials and products. Among all substances, lead and mercury are of particular focus, due to their well-known toxicity. In the field of energetic materials, lead- and mercury-based salts have been extensively used as primary explosives [2-4]. Therefore, the replacement of these energetic materials is of outstanding importance. Energetic materials are undoubtedly used in military applications; however, pyrotechnic compositions or explosives in general are also frequently used in many civilian domains, such as fireworks, mining, the automotive industry, and the aerospace industry, strengthening the need to find alternative materials. The development of "green" energetic materials has consequently caused intense interest among the scientific community [5-7]. However, this novel research topic must not neglect the safety of energetic materials regarding production, handling and transport. For example, mechanical, electrostatic and thermal stresses can accidentally initiate dramatic or hazardous reactions in such compounds. For this reason, international standards have been established to govern the safe transport of energetic materials [8]. Finally, the development of safer and green energetic materials that retain their reactive properties is a tremendous issue for the industry, with a focus on enabling new possibilities in civilian, military and space pyrotechnic applications. A promising potential candidate to successfully address the aforementioned points could be a class of energetic composites named "thermites" or "Metastable Intermolecular Composites" (MICs) [9]. These energetic composite materials, featuring homogeneous physical mixtures of metal fuel $(\mathrm{Al}, \mathrm{Mg}, \ldots)$ and oxidiser $\left(\mathrm{WO}_{3}, \mathrm{MoO}_{3}, \ldots\right)$ at the microscale, undergo a highly exothermic redox reaction leading to temperatures above $2000^{\circ} \mathrm{C}[10]$. Some of these materials may exhibit higher densities and energy amount in volume than traditional organic 
explosive molecules. In addition, if nanostructured powders are used, the pyrotechnic performance of such composites is greatly improved [11], such as an easier ignition and combustion velocities as high as $2500 \mathrm{~m} / \mathrm{s}$ for $\mathrm{Al} / \mathrm{Bi}_{2} \mathrm{O}_{3}$ [12] or $\mathrm{Al} / \mathrm{CuO}$ [13] compositions. As a reminder, the explosive rates of the $\mathrm{Hg}$ - and $\mathrm{Pb}$-based primary explosives are within the 4000 to $5000 \mathrm{~m} / \mathrm{s}$ range. However, energetic nanocomposites are often more sensitive than their micron-sized counterparts. For example, this material exhibits an increased sensitivity to mechanical and electrostatic stimuli, making the handling of the material hazardous. In the literature, the desensitisation of nanothermites is an issue that can be successfully addressed by using mainly carbonaceous compounds or a Viton fluoropolymer as additives [14-19]. Unfortunately, by adding these desensitisers, the pyrotechnic properties of the energetic composites are often drastically altered $[16,18]$.

Here, we report the use of an organic conducting polymer as an additive within the $\mathrm{Al} / \mathrm{WO}_{3}$ pyrotechnic formulation. Among the family of intrinsic conducting polymers, polyaniline (PAni) was selected due to its low cost and simple synthesis, environmental stability, tuneable electrical conductivity $[20,21]$ and lubricating properties [22]. The organic additive was synthesised for different aspect ratios and was added to the selected nanothermite, whose pyrotechnic performance was precisely characterised.

\section{Experimental section}

Chemical reagents were used "as received" and without any further purification. Aniline $\left(\mathrm{C}_{6} \mathrm{H}_{5} \mathrm{NH}_{2}, 99 \%\right)$, potassium hydrogen diiodate $\left(\mathrm{KH}\left(\mathrm{IO}_{3}\right)_{2}, 99.8 \%\right)$, sodium hypochlorite ( NaOCl, available chlorine 4-4.99\%), acetonitrile $\left(\mathrm{CH}_{3} \mathrm{CN}, 99 \%\right)$ and polyaniline (Emeraldine salt, $\mathrm{M}_{\mathrm{w}}>15,000$ g.mol${ }^{-1}, \sigma=2-4{\mathrm{~S} . \mathrm{cm}^{-1}}^{-1}$ were purchased from Sigma-Aldrich. Hydrochloric acid $(\mathrm{HCl}, 37 \%)$ was obtained from Prolabo. Aluminium (Al, Al-50-P, SSA $\left.=37 \mathrm{~m}^{2} \cdot \mathrm{g}^{-1}\right)$ and 
tungsten trioxide $\left(\mathrm{WO}_{3}, \mathrm{SSA}=13.5 \mathrm{~m}^{2} \cdot \mathrm{g}^{-1}, 99.9 \%\right)$ nanopowders were provided by NovaCentrix (United States) and DK Nanomaterials (China), respectively. Polyaniline nanofibre synthesis:

The protocol is inspired by the work of Rahy et al. $[23,24]$ and can be described as follows: 1 $\mathrm{mL}$ of aniline was mechanically stirred for $20 \mathrm{~min}$ in $100 \mathrm{ml} \mathrm{HCl}(1 \mathrm{M})$. Next, $100 \mathrm{ml}$ of aqueous solution containing $0.4864 \mathrm{~g}$ of $\mathrm{KH}\left(\mathrm{IO}_{3}\right)_{2}$ was added to the aniline solution, and after $5 \mathrm{~min}, 5 \mathrm{~mL}$ of $\mathrm{NaOCl}$ was added dropwise to the previous solution while keeping it under vigorous stirring. After complete introduction, the stirring was stopped and the final solution was kept at room temperature for either 0.3 hours or 72 hours. Solid products were recovered by centrifugation and then washed with $\mathrm{HCl}(1 \mathrm{M})$, methanol and acetone. Consecutive overnight drying at $60{ }^{\circ} \mathrm{C}$ was performed to obtain the final powders. The "as-prepared" materials were labelled "PAni_SF" and "PAni_LF" for the samples obtained after a short (0.3 h) and a long ageing time $(72 \mathrm{~h})$, respectively. A commercial polyaniline reagent, PAni_Com, was also selected to carry out the investigation.

\section{Nanothermite preparation:}

The energetic mixtures were prepared by combining aluminium $(\mathrm{Al})$, tungsten trioxide $\left(\mathrm{WO}_{3}\right)$ and conducting polymer (PAni, 0-5wt \%) nanopowders in predefined proportions in acetonitrile. Note that the Al-50-P powder is made of aluminium nanoparticles that exhibit a core-shell structure, i.e., an $\mathrm{Al}$ metallic core surrounded by an $\mathrm{Al}_{2} \mathrm{O}_{3}$ layer. The active $\mathrm{Al}$ content in the powdered fuel was determined to be equal to $57.9 \mathrm{wt} \%$. To boost the energetic performance of the composites investigated, an excess of fuel, compared to the following stoichiometric chemical reaction (1), was considered.

$$
2 \mathrm{Al}_{(\mathrm{s})}+\mathrm{WO}_{3(\mathrm{~s})} \rightarrow \mathrm{Al}_{2} \mathrm{O}_{3(\mathrm{~s})}+\mathrm{W}_{(\mathrm{s})}
$$

By taking the excess into account, the amount of active aluminium was calculated according to the equivalence ratio $(\Phi)$ described in Eq. (2) and fixed to a value of 1.4: 


$$
\Phi=\frac{\left(\frac{F}{O}\right) \exp }{\left(\frac{F}{O}\right) s t}
$$

where $F$ and $O$ are the mass ratio of fuel (Al) to oxidiser $\left(\mathrm{WO}_{3}\right)$. The subscripts exp. and st. stand for the experimental and stoichiometric ratios.

The resulting dispersions (with or without additive) were homogenised for 1 hour by alternating stirring and ultrasonication (sonotrode, $300 \mathrm{~W}, 40 \mathrm{kHz}$, pulse of $0.25 \mathrm{~s}$ ). Finally, the solvent was evaporated under 200 mbar and $80^{\circ} \mathrm{C}$ to obtain dry grey compositions. The diverse energetic composites were labelled “AW_PAni_xy”. A, W, x, and y represent the fuel, the oxidiser, the nature of polyaniline (SF, LF, or com.), and the amount of polyaniline, respectively.

\section{Characterisation techniques:}

Fourier Transform Infrared (FT-IR) spectroscopic analysis of the polyaniline samples was conducted on a Bruker Tensor 27 spectrometer in transmission mode ( $\mathrm{KBr}$ disks). The spectra were recorded within a $4000-520 \mathrm{~cm}^{-1}$ range (resolution of $4 \mathrm{~cm}^{-1}$ ) by superposing 16 scans. The elemental analyses $(\mathrm{C}, \mathrm{N}, \mathrm{H}$ and $\mathrm{O})$ of the polyaniline samples were performed on a Thermo Finnigan Flash EA 1112 apparatus at $1050^{\circ} \mathrm{C}$ supported by a helium/oxygen flow. Reaction products $\left(\mathrm{CO}_{2}, \mathrm{H}_{2} \mathrm{O}\right.$, and $\left.\mathrm{NO}_{\mathrm{x}}\right)$ were separated on a chromatography column and quantified by using a thermal conductivity detector. The amount of chlorine was calculated with respect to $100 \%$ of the material. The morphology and microstructure of the different materials (polyaniline, oxidiser and fuel) were observed via Transmission and Scanning Electron Microscopy (TEM, SEM) by using a CM200 microscope (Philips, $200 \mathrm{kV}$ ) and a DSM 982 Gemini (Zeiss, $10 \mathrm{kV}$ ) microscope, respectively.

Sensitivity tests: for the impact, friction and electrostatic discharge sensitivity measurements, the threshold determined on the loose powder is the highest value, for which 6 reaction tests are related to a no-combustion result. The probability of obtaining no combustion at all is 
$98.4 \%$ at that threshold value. All of the experiments were performed in compliance with the NATO standards [25-27].

The impact sensitivity is determined by a Julius Peters BAM Fallhammer apparatus. During the experiment, the energetic material is placed between two steel cylinders. A hammer ( 1 or $5 \mathrm{~kg}$ ) falls from various drop heights (max. of $1 \mathrm{~m}$ ) onto the energetic material. The impact threshold value is given in joules $(\mathrm{J})$. The friction sensitivity is determined by a BAM friction apparatus. Under the experimental conditions, the energetic material is placed between a ceramic plate and a peg: the force applied is a function of the weights fixed at specific locations on a lever arm. The friction threshold value is given in Newtons $(\mathrm{N})$. The electrostatic discharge (ESD) sensitivity is measured by using an ESD 2008 testing device from OZM Research s.r.o., Czech Republic, with a discharge voltage of 4-10 kV and a distance of $1.0 \mathrm{~mm}$ between the electrodes. The ESD threshold value is given in joules (J). Reactivity tests: a schematic of the equipment used to ignite the formulations and to record the combustion phenomena is shown in Fig. 1. Typically, nanothermite powders were loosely packed in cylindrical glass tubes with an inner diameter of $3 \mathrm{~mm}$ and a length of $150 \mathrm{~mm}$. An electric primer was used for the ignition of the nanothermites, as proposed by Comet et al. [28]. The combustion behaviours of the energetic compositions (with or w/o polyaniline) were recorded by using a Photrom FASTCAM high-speed camera $\left(5 \times 10^{4}\right.$ frames/s $)$ placed perpendicularly to the glass tubes. The combustion velocities were calculated from a series of four tests. 


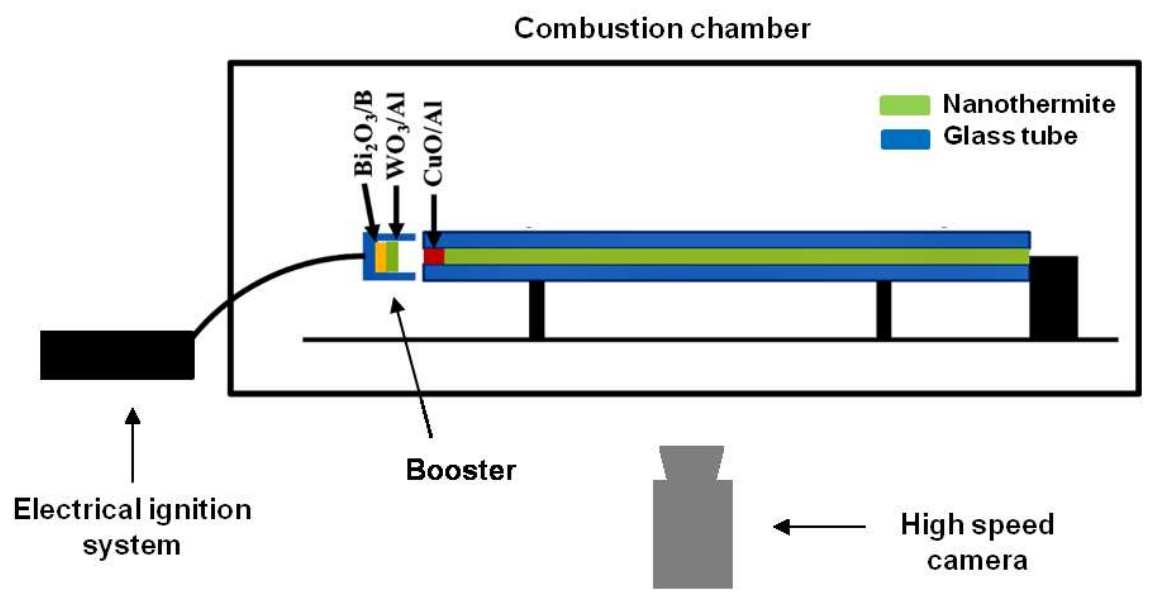

Figure 1: Schematic of the reactivity testing equipment used for nanothermites.

The apparent densities of the nanothermites tested were kept constant at approximately $10 \%$

$(\approx$ loose powder) of their corresponding theoretical maximum densities (TMD) calculated from Eq. (3).

$$
T M D=1 / \sum_{i=1}^{n} \frac{m i}{\rho i}
$$

where $i$ is the considered chemical species and $m$ and $\rho$ are its mass and density, respectively.

\section{Results and Discussion}

\section{Characterisation of the PAni conducting polymer}

The samples recovered at the end of the synthesis procedure were a dark-green colour, and the yields were calculated to range from $21 \%$ to $47 \%$ for synthesis times ranging from 0.3 to $72 \mathrm{~h}$. This specific colour characterises the conducting phase of the PAni-named emeraldine salt, as opposed to the insulating leucoemeraldine and pernigraniline phases. The molecular structure of the emeraldine base is shown in Figure 2, where two chemical entities called benzenoid and quinoid coexist in equimolar amounts. The partial protonation of the quinoid functional group leads to the green emeraldine salt. 


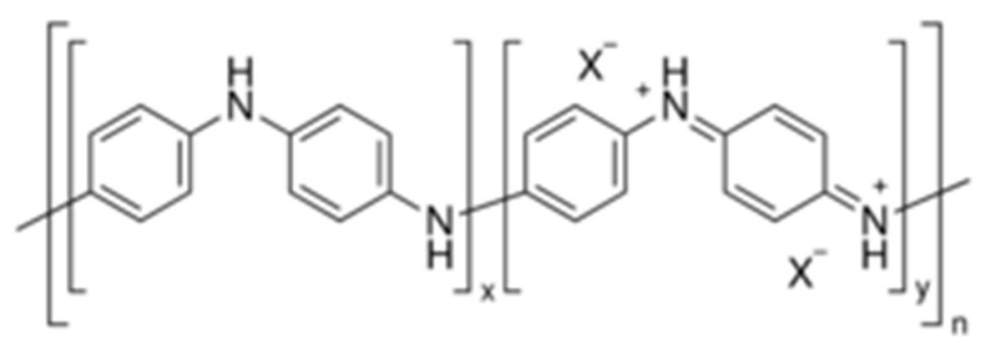

Figure 2: Molecular structure of the conducting emeraldine salt form $(X=$ anion).

The FTIR spectroscopic analysis of each "as-prepared" PAni sample is presented in Fig. 3.

The FTIR spectra of PAni-com, PAni_SF, and PAni_LF are consistent with the literature [23$24]$ and are characterised by two active domains in the ranges of $1600-1100 \mathrm{~cm}^{-1}$ and $900-400$ $\mathrm{cm}^{-1}$. The vibration bands located at $1560 \mathrm{~cm}^{-1}$ and $1488 \mathrm{~cm}^{-1}$ were attributed to the stretching $(v)$ modes of the quinoid and benzenoid units, respectively. The latter entity confirms the successful synthesis of the conducting PAni polymer. The formation of such a molecular structure was further strengthened by the infrared band at $1250 \mathrm{~cm}^{-1}$ relating to the $v(\mathrm{C}-\mathrm{N}+)$ in the polaron lattice of the PAni polymer. The bands at $1300 \mathrm{~cm}^{-1}$ and $1146 \mathrm{~cm}^{-1}$ were identified as originating from the $(\mathrm{C}-\mathrm{N})$ stretching $(v)$ and $(\mathrm{C}-\mathrm{H})$ bending $(\delta)$ modes in a benzenoid and a quinoid unit, respectively. Finally, in the lowest wavenumber domain, the bands at $823 \mathrm{~cm}^{-1}$ and $504 \mathrm{~cm}^{-1}$ were assigned to the bending $(\delta)$ vibration of the $\mathrm{C}-\mathrm{H}$ bond inside a 1,4-disubstituted aromatic cycle. 


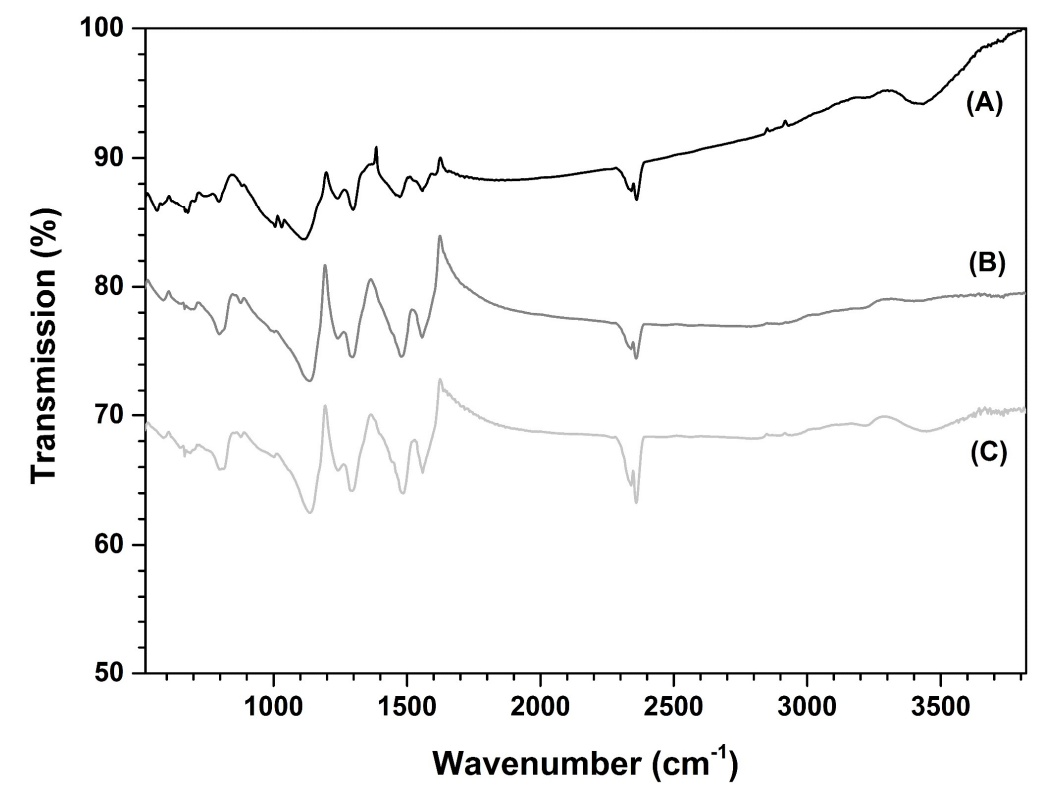

Figure 3: FTIR spectra of the PAni_com (A), PAni_SF (B) and PAni_LF (C) samples. The spectra were shifted to a better legibility.

The data relating to the elemental analyses performed on each of the three polymer samples are presented in Table 1. The amount of each chemical element is similar for the PAni_SF and PAni_LF samples, suggesting that the specific ageing time does not affect the chemical composition of the synthesised materials. The main difference between the commercial sample (PAni_com) and the synthesised ones is that the amount of oxygen is higher in the former case, which may be explained by the fact that the protonation of the commercial product is achieved by means of a sulfonic acid $\left(\mathrm{RSO}_{3} \mathrm{H}\right.$, where $\mathrm{R}$ is an alkyl group) according to the manufacturer's data. Hydrochloric acid $(\mathrm{HCl})$ was used for PAni_SF and PAni_LF. 


\begin{tabular}{|ccccccc|}
\hline & $\mathbf{\% C}$ & $\mathbf{\%} \mathbf{N}$ & $\mathbf{\% H}$ & $\mathbf{\% O}$ & $\mathbf{\% S}$ & $\mathbf{\% C l}$ \\
\hline PAni_com & 61.15 & 6.31 & 5.65 & 15.77 & 7.99 & $/$ \\
\hline PAni_SF & 52.33 & 10.39 & 4.21 & 10.91 & $/$ & 22.16 \\
\hline PAni_LF & 53.59 & 9.48 & 3.88 & 10.09 & $/$ & 22.96 \\
\hline
\end{tabular}

Table 1: Chemical composition of the different polyaniline samples by elemental analysis. The chlorine amount was calculated with respect to $100 \%$ of the material.

Scanning and transmission electron microscopy (SEM and TEM) images of the PAni samples are presented in Figure 5. Whereas highly agglomerated 500-nm-sized sphere-like particles are observed for the commercial sample (PAni_com, Fig. 4A), the "as-synthesised" PAni polymers (PAni_SF and PAni_LF) exhibit a nanoscale-fibre morphology, as expected from the works of Rahy [23-24]. Polymerisation times of 0.3 h (Fig. 4B) and 72 h (Fig. 4C) lead to the formation of agglomerates of short nanofibres (length $<0.5 \mu \mathrm{m}$ ) and more isolated long nanofibres (length $=1-2 \mu \mathrm{m})$, respectively. The average diameter is close to $50 \mathrm{~nm}(\varnothing)$ and is independent of the polymerization time. 

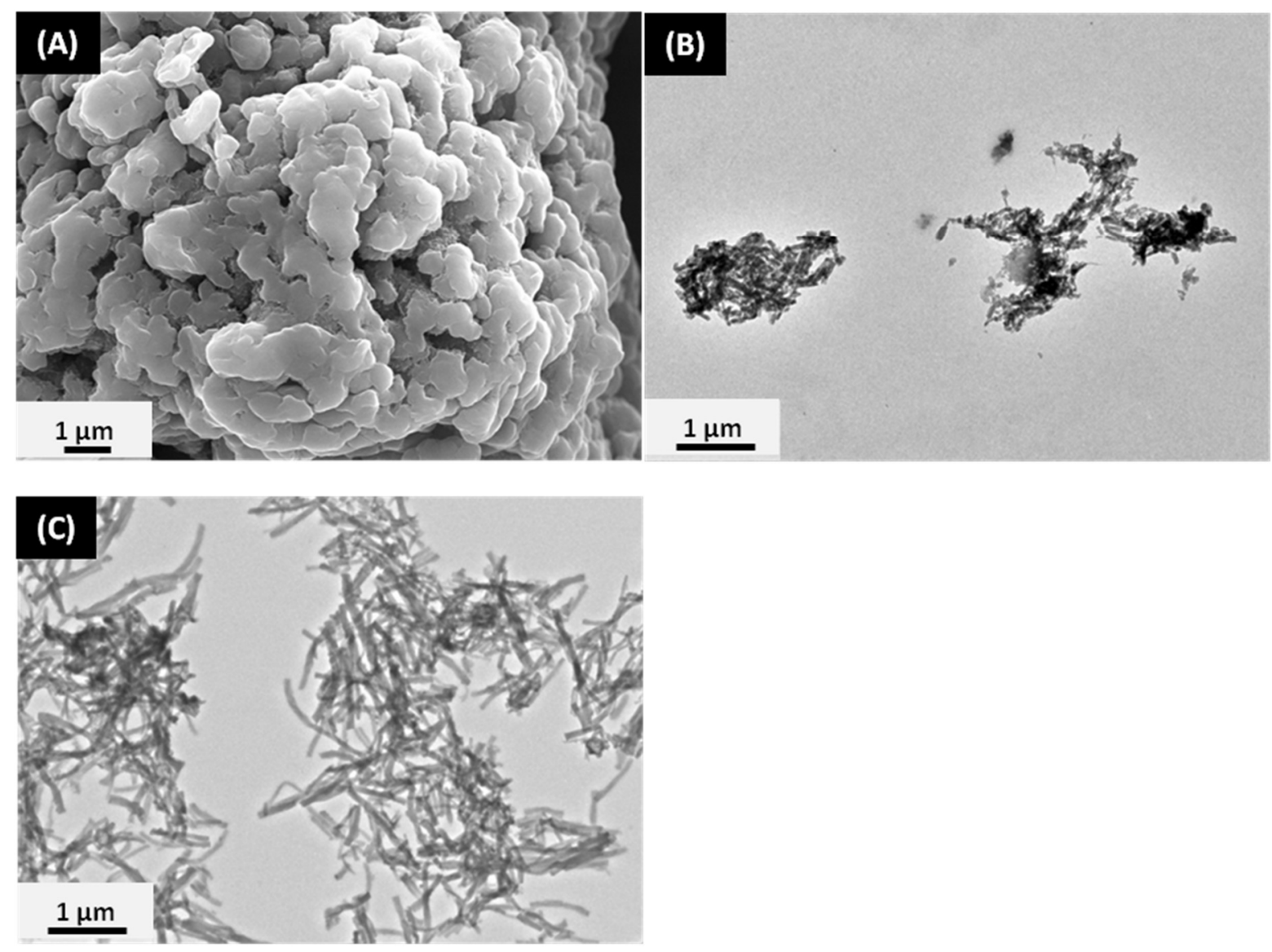

Figure 4: SEM and TEM images of the PAni_com (A), PAni_SF (B) and PAni_LF (C) conducting polymers.

\section{Characterisation of the PAni-based Al/WO 3 nanothermites}

One of the fundamental aspects relating to a mixture of several components concerns the distribution of each individual element within the whole volume. A poor dispersion of the different elements does not reach the optimised performance of the mixture investigated, whereas a good distribution does. Therefore, the above-described experimental procedure was optimised to ensure a good homogeneity between aluminium, tungsten (VI) oxide and the conducting PAni polymer, especially if a maximum of $5 \mathrm{wt} \%$ of the PAni component is present in the $\mathrm{Al} / \mathrm{WO}_{3}$ energetic mixture. To control the homogeneity of the polyaniline-based $\mathrm{Al} / \mathrm{WO}_{3}$ nanothermites, meticulous observations with the TEM apparatus were systematically 
conducted. Representative images of the four energetic systems (one without PAni and three with 5 wt $\%$ of PAni_com, PAni_SF, and PAni_LF) are shown in Figure 5.
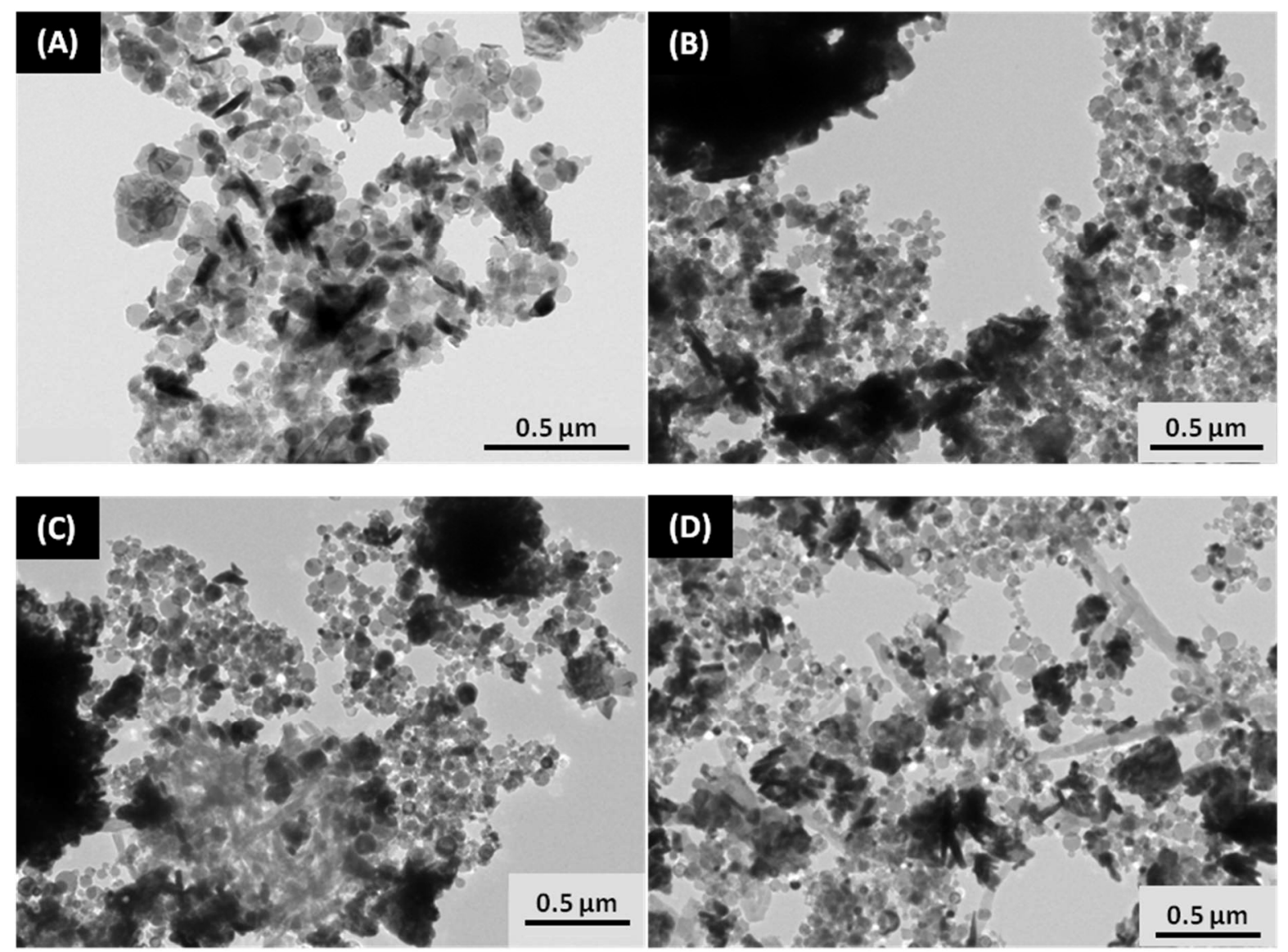

Figure 5: TEM images of the energetic systems of $\mathrm{Al} / \mathrm{WO}_{3}(\mathrm{~A}), \mathrm{Al} / \mathrm{WO}_{3} / \mathrm{PAni}$-com (B), Al/WO $3 / \mathrm{PAni}_{3} \mathrm{SF}$ (C) and Al/WO $3 /$ PAni_LF (D).

The binary $\mathrm{Al} / \mathrm{WO}_{3}$ nanothermite (Figure $5 \mathrm{~A}$ ) is characterised by a homogeneous distribution, at the nanoscale, of spherical aluminium nanoparticles and tungsten (VI) oxide platelets. No $\mathrm{WO}_{3}$ - or Al-rich domains were noted within the mixture from the observation of the TEM images. Regarding the PAni-based nanothermites, two distinct characteristics were identified: first, a ternary system with the presence of PAni aggregates in a well-distributed $\mathrm{Al} / \mathrm{WO}_{3}$ mixture obtained by adding commercial fibres (PAni_com, Fig. 6B) and short fibres (SF_PAni, Fig. 6C) of the conducting polymer; and second, a ternary system that is much more homogeneous with respect to the volume distribution of the components when adding 
long nanofibres of polyaniline (PAni_LF, Fig. 6D). Furthermore, the individual sizes of the PAni aggregates in $\mathrm{Al} / \mathrm{WO}_{3} / \mathrm{PAni}$ _com are larger compared to those within the $\mathrm{Al} / \mathrm{WO}_{3} / \mathrm{PAni}$ SF formulations. This observation is consistent with the TEM analyses conducted on the single conducting polymer (Fig. 5), where agglomeration is detected for the PANi_com and the PAni_SF polymers.

\section{Sensitivities of PAni-doped $\mathrm{Al} / \mathrm{WO}_{3}$ nanothermites}

The electrical and mechanical sensitivities of the binary and ternary energetic formulations (nanothermites) are presented in Table 2. The sensitivities were determined according to the protocol detailed in the Experimental Section.

\begin{tabular}{|cccc|}
\hline & ESD (mJ) & Friction (N) & Impact (J) \\
\hline Standards & $\mathbf{1 0 0}$ & $\mathbf{8 0}$ & $\mathbf{2}$ \\
\hline Al/WO3 & $<0.14$ & 8 & $>50$ \\
\hline Al/WO3/PAni_com & $<0.14$ & 192 & 45 \\
\hline Al/WO3/PAni_SF & 0.14 & 288 & 10 \\
\hline Al/WO3/PAni_LF & 120 & $>360$ & 20 \\
\hline
\end{tabular}

Table 2: Sensitivity thresholds for $\mathrm{Al} / \mathrm{WO}_{3}$ nanothermites mixed with the PAni polymer $(5 \mathrm{wt} \%$, $\mathrm{com}=$ commercial, $\mathrm{SF}=$ short nanofibres, $\mathrm{LF}=$ long nanofibres); ESD for ElectroStatic Discharge.

With regard to the electrostatic discharge sensitivity (ESD), the use of the commercial conducting polyaniline or "as-synthesised" short nanofibres as desensitising additives does not change the sensitivity threshold of the binary $\mathrm{Al} / \mathrm{WO}_{3}$ nanothermite, which is taken as reference. In this context, a constant value of $0.14 \mathrm{~mJ}$ was determined, which is well below the standard value $(>100 \mathrm{~mJ})$. At this level, an accidental decomposition reaction can occur, for example, during handling by an operator, and consequently presents a considerable 
hazard. However, when long nanofibres of polyaniline (PAni_LF) were mixed with the $\mathrm{Al} / \mathrm{WO}_{3}$ energetic formulation, a drastic change occurred, leading to an increase in the ESD thresholds from below $0.14 \mathrm{~mJ}$ to $120 \mathrm{~mJ}$. As the electrical conductivity values of each PAni sample are similar, the behaviour of the $\mathrm{Al} / \mathrm{WO}_{3} / \mathrm{PAni} \_\mathrm{LF}$ nanothermites toward the ESD sensitivity may be explained in terms of the preparation of the energetic formulations and the percolation theory. First, as previously demonstrated, the PAni_LF sample is much less agglomerated (Fig. 5) and better dispersed in the $\mathrm{Al} / \mathrm{WO}_{3}$ energetic formulation than its PAni_com and PAni_SF counterparts (Fig. 6). This aspect is correlated with the fact that PAni_LF exhibits the highest aspect ratio of the nanofibres (length/diameter); thus, we think that the percolation of the polymer additive is effective in this case for a $5 \mathrm{wt} \%$ content $\approx \approx 15$ vol. \%). This $\mathrm{Al} / \mathrm{WO}_{3} / \mathrm{PAni}$ LLF nanothermite can be described as an $\mathrm{Al} / \mathrm{WO}_{3}$ mixture embedded in a three-dimensional PAni conducting network. This conducting network is able to evacuate electrostatic charges through the composites, thus retaining little energy to heat the energetic formulation and to lead to its ignition. For the other two PAni samples (PAni_SF, PAni_com), a more or less high level of aggregation and smaller aspect ratios exclude the use of the percolation theory. Table 3 shows the influence of the mass content of long conducting nanofibres on the ESD sensitivity of a binary $\mathrm{Al} / \mathrm{WO}_{3}$ nanothermite. It can be seen that at $3 \mathrm{wt} \%$, the threshold value decreases until it is below $0.14 \mathrm{~mJ}$, suggesting that the percolation is reached between $3-5 \mathrm{wt} \%$ by long PAni nanofibres $(\approx 10-15$ vol.\%). This result is consistent with the work of Steelman et al. [16]. In that paper, 13 vol\% of carbon nanotube (CNT) additive, exhibiting a similar morphology and size compared to the polyaniline nanofibres, was identified as the specific percolation threshold within the $\mathrm{Al} / \mathrm{CuO}$ nanocomposites.

With regard to the friction sensitivity, the binary $\mathrm{WO}_{3} / \mathrm{Al}$ nanothermite is highly sensitive, with a threshold value of $8 \mathrm{~N}$ compared with the standard value of $80 \mathrm{~N}$ for the transport of 
explosive goods [8]. The use of the conducting polyaniline, regardless of the form it takes (PAni_com, PAni_SF, and PAni_LF at 5 wt \%), leads to a gradual increase in the sensitivity threshold value of the nanothermite up to a maximum of $360 \mathrm{~N}$ (i.e., largely above the standard value and therefore characterising the energetic formulation as totally insensitive). The insensitive character of the different $\mathrm{Al} / \mathrm{WO}_{3} /$ polymer formulations might be explained by the lubricating properties of polyaniline [22]. This mechanical behaviour might result from a low interchain cross-linking rate or weak interchain interactions (e.g., hydrogen bonding or $\pi-\pi$ stacking), which allows the sliding of polymeric chains with respect to each other during mechanical stresses, thereby absorbing a part of the energy from stimuli. The non-absorbed energy is then insufficient to ignite the $\mathrm{Al} / \mathrm{WO}_{3}$ energetic nanocomposite. However, as seen, the insensitivity increases as follows: PAni_com $<$ PAni_SF $<$ PAni_LF. The latter observation can be correlated with the homogeneity of the $\mathrm{Al} / \mathrm{WO}_{3} /$ conducting polymer energetic formulation (Fig. 6): the higher the homogenisation of the $\mathrm{Al} / \mathrm{WO}_{3} /$ polymer is, the lower the friction sensitivity becomes. Due to the higher effect observed with the long nanofibre polyaniline, the amount of mass of this polymer within the formulation was investigated (Table 3). By reducing the mass of long nanofibres inside the $\mathrm{Al} / \mathrm{WO}_{3}$ nanothermite, a downward trend could be observed, together with a threshold value remaining above the standard value $(84 \mathrm{~N}$ at $1 \mathrm{wt} \%$ vs. $80 \mathrm{~N})$ and 10 times higher than the friction sensitivity of the PAni-undoped $\mathrm{Al} / \mathrm{WO}_{3}$ nanothermite $(8 \mathrm{~N})$.

Regarding the sensitivity to impact, the $\mathrm{Al} / \mathrm{WO}_{3}$ binary formulation can be considered to be insensitive, with a threshold value higher than $50 \mathrm{~N}$, if we refer to the standard set at $2 \mathrm{~J}$ by the United Nations [8]. The use of polyaniline as an additive leads to a systematic decrease in ignition energy down to a minimum value of $10 \mathrm{~J}$ for the shortest polyaniline nanofibres; however, in all cases, the ternary nanothermites $\left(\mathrm{A} 1 / \mathrm{WO}_{3}\right.$ with PAni_com, PAni_SF, or PAni_LF) remain safe with respect to the standard, which can clearly be interpreted as a 
success. As reported in the literature $[30,31]$, the ignition of nanothermites can be activated by the formation of hot spots inside the investigated composite subjected to stress. Hot spots can be generated in three distinct ways:

. adiabatic compression of gases trapped inside the pores within the composite, . chemical reactions due to particles that crash on the move, . interparticulate friction.

As the friction sensitivity of polyaniline-doped nanocomposites is low (i.e., $>190 \mathrm{~N}$ vs. $8 \mathrm{~N}$ for an $\mathrm{Al} / \mathrm{WO}_{3}$ system) the last hypothesis can reasonably be discarded. Regarding the second hypothesis, it could be validated if the additive presented higher mechanical properties than the hardest components composing the conventional energetic mixtures $\left(\mathrm{Al}_{2} \mathrm{O}_{3}\right.$ and $\mathrm{WO}_{3}$ ceramics); this is not expected in the present case, as the additive belongs to the polymer family. Consequently, the hot spot formation probably results from the adiabatic compression of gases inside the material during the impact stress. As the $\mathrm{Al} / \mathrm{WO}_{3}$ energetic nanothermite is a very low gas-generating composite, only the gasification of the polyaniline particles can back up this hypothesis. From thermogravimetric analyses under oxidising atmosphere (not shown here), the combustion of polyaniline starts at $250^{\circ} \mathrm{C}$ and ends at $300^{\circ} \mathrm{C}$ and $600^{\circ} \mathrm{C}$ for the nanofibres (PAni_SF \& PAni_LF) and the micron-sized spherical particles (PAni_com), respectively. The high agglomeration and low aspect ratio of the micron-sized particles might be responsible for the slower combustion kinetics. Because the gasification of the largest polyaniline particles is more difficult, the $\mathrm{Al} / \mathrm{WO}_{3} / \mathrm{PAni}$ com is less sensitive to impact (difficult hot spot formation) than its $\mathrm{Al} / \mathrm{WO}_{3} / \mathrm{PAni} \_\mathrm{SF}$ and $\mathrm{Al} / \mathrm{WO}_{3} / \mathrm{PAni} \mathrm{LF}$ counterparts (45 J vs. $10 \mathrm{~J}$ and $20 \mathrm{~J}$, respectively).

By decreasing the mass content of the long conducting nanofibres (Table 3), the impact threshold increases to reach the value of the binary $\mathrm{Al} / \mathrm{WO}_{3}$ nanothermite, thus confirming the previously given explanations. 


\begin{tabular}{|c|c|c|c|c|}
\hline & $\begin{array}{c}\text { Weight } \% \text { of } \\
\text { conducting polymer }\end{array}$ & $\operatorname{ESD}(\mathbf{m J})$ & Friction (N) & $\operatorname{Impact}(J)$ \\
\hline Standards & l & 100 & 80 & 2 \\
\hline \multirow{4}{*}{ PAni_LF } & 5 & 120 & $>360$ & 20 \\
\hline & 3 & 0.14 & 108 & 45 \\
\hline & 1 & $<0.14$ & 84 & $>50$ \\
\hline & 0 & $<0.14$ & 8 & $>50$ \\
\hline
\end{tabular}

Table 3: Sensitivity thresholds for an $\mathrm{Al}_{\text {/ }} \mathrm{WO}_{3}$ nanothermite mixed with PAni long nanofibres (PAni_LF).

\section{Combustion performance of PAni-doped $\mathrm{Al} / \mathrm{WO}_{3}$ nanothermites}

In this section, the combustion performance of the $\mathrm{Al} / \mathrm{WO}_{3} / \mathrm{PAni} \mathrm{LF}$ nanothermite $(5 \mathrm{wt} \%)$ is compared with the conventional $\mathrm{Al} / \mathrm{WO}_{3}$ nanothermite performance. It appears as the only ternary $\mathrm{Al} / \mathrm{WO}_{3} /$ conducting polymer energetic formulation that exhibits complete insensitivity to mechanical and electrical stresses, in accordance with the NATO standard (Table 3).

The combustion energies of the two energetic nanocomposites were measured by using a calorimetric bomb. The released energy of the $\mathrm{Al} / \mathrm{WO}_{3}$ was determined to be close to 2815

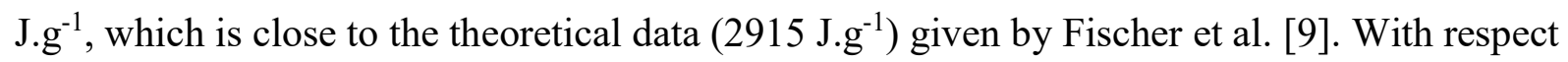
to the PAni-doped $\mathrm{Al} / \mathrm{WO}_{3}$ nanocomposites, a lower value was found to be equal to $2536 \mathrm{~J} . \mathrm{g}^{-}$ 1, suggesting a less powerful energetic character when the desensitising agent is added into the reference binary composites.

Figure 6 shows some selected video frames of the combustion of these two energetic composites. Combustion tests were performed in glass tubes filled with loose powders at loading densities calculated to be equal to $0.39 \pm 0.02 \mathrm{~g} . \mathrm{cm}^{-3}$ and $0.43 \pm 0.02 \mathrm{~g} . \mathrm{cm}^{-3}$, corresponding to $8.2 \%$ and $9.1 \%$ of the TMD of the $\mathrm{Al} / \mathrm{WO}_{3}$ and the $\mathrm{Al} / \mathrm{WO}_{3} / \mathrm{PAni} \_\mathrm{LF}$ energetic nanocomposites, respectively. From a meticulous analysis of the video sequences, 
dark zones can be visualised (i.e., at the end of the combustion for the $\mathrm{Al} / \mathrm{WO}_{3}$ nanothermite $(0.5 \mathrm{~ms})$ and directly from the ignition for the $\mathrm{Al} / \mathrm{WO}_{3} / \mathrm{PAni} \_\mathrm{LF}$ nanothermite $\left.(0.4 \mathrm{~ms})\right)$.

Whereas in the former case, these zones exhibit a classical decrease in temperature after the composition front has passed, in the latter case, these zones also suggest the spatial positioning of the conducting polymer in the ternary composition because the combustion of polyaniline requires energy and consequently accelerates cooling. From this consideration, a homogeneous distribution of PAni can be observed over the whole length of the tube (Figure 6 B, time: $0.5 \mathrm{~ms})$.

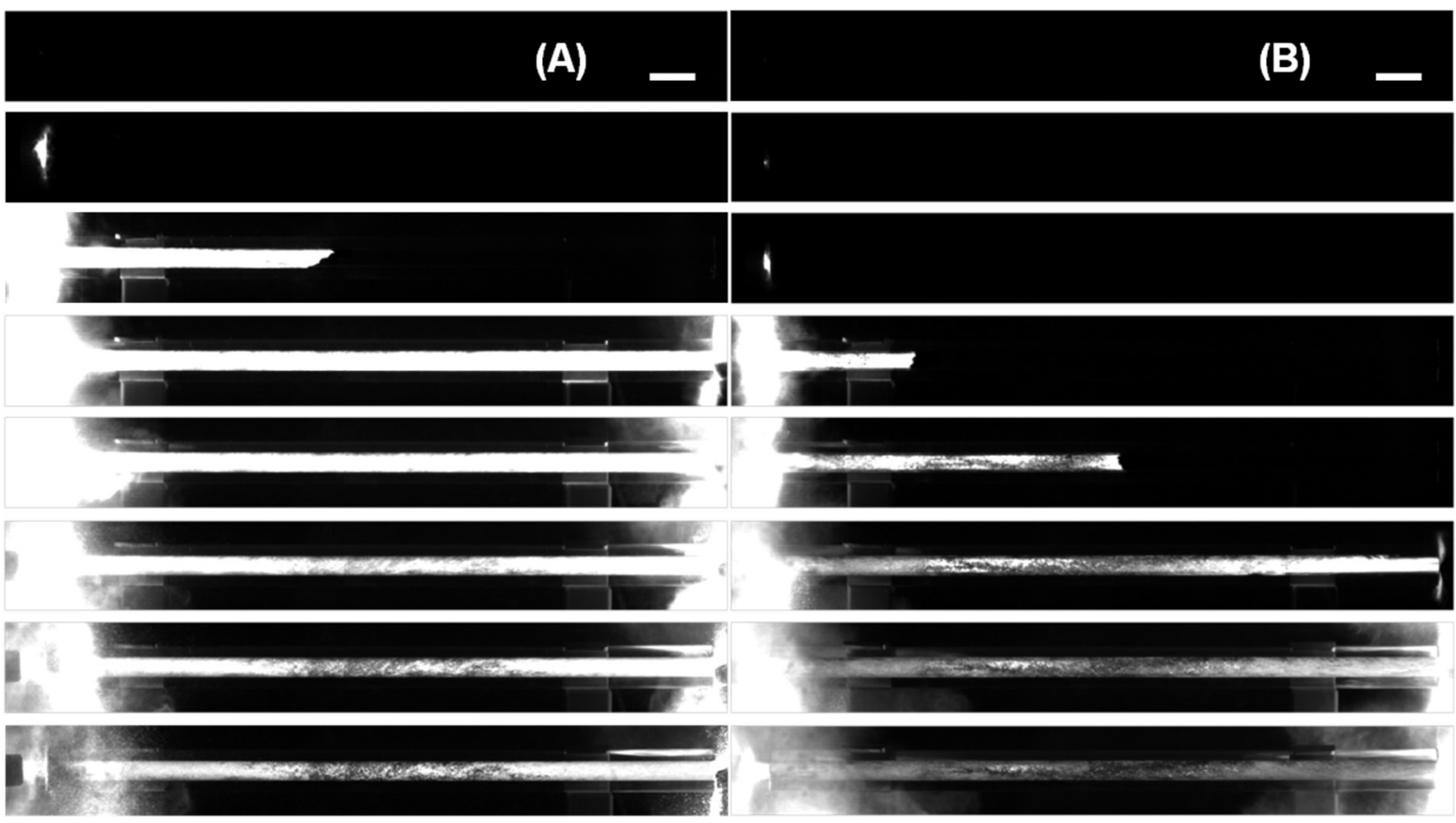

Figure 6: Sequences of combustion images of the $\mathrm{Al} / \mathrm{WO}_{3}(\mathrm{~A})$ and the $\mathrm{Al} / \mathrm{WO}_{3} / \mathrm{PAni}_{-} \mathrm{LF}(\mathrm{B})$ energetic nanocomposites. The scale bar (in the inset of each first image) represents $10 \mathrm{~mm}$, and the time scale ranges from 0 to $0.7 \mathrm{~ms}$ (the step size is $0.1 \mathrm{~ms})$.

The progress of the combustion front filmed through the tube, for each energetic composite, is plotted as a function of time in Figure 7. Different profiles can be divided into three and four zones for the $\mathrm{Al} / \mathrm{WO}_{3}$ and the $\mathrm{Al} / \mathrm{WO}_{3} / \mathrm{PAni} \_\mathrm{LF}$ composites, respectively. The combustion regimes are multistage, and based on our experience and knowledge [18, 32-33], an attempt to 
explain them is described below. The first millimetres of the tubes (length $<5 \mathrm{~mm}$ ) characterise the combustion of the $\mathrm{Al} / \mathrm{CuO}$ igniting composition; consequently, no signals were intentionally taken into account. Within zone 1, a displacement of the reaction front was recorded as a function of time; this displacement represents the combustion of the $\mathrm{Al} / \mathrm{WO}_{3}$ investigated thermite (w/o and with polyaniline). Nevertheless, at this stage, an influence of the CuO-based igniting composition (diffusion/conduction phenomena) cannot be reasonably neglected. The second zone is attributed to the self-combustion of the energetic formulations investigated and to combustion velocities of $620 \pm 5 \mathrm{~m} / \mathrm{s}$ and $256 \pm 14 \mathrm{~m} / \mathrm{s}$, which were calculated for the $\mathrm{Al} / \mathrm{WO}_{3}$ and the $\mathrm{Al} / \mathrm{WO}_{3} / \mathrm{PAni} \mathrm{LF}$ nanocomposites, respectively. Finally, in the last millimetres of the glass tubes (zone 3), an acceleration of the combustion phenomenon is observed for the PAni-doped nanocomposites. The resulting combustion velocity was calculated to be close to $414 \pm 35 \mathrm{~m} / \mathrm{s}$ and was referenced as the actual combustion velocity of the $\mathrm{Al} / \mathrm{WO}_{3} / \mathrm{PAni} \_\mathrm{LF}$ energetic formulation.

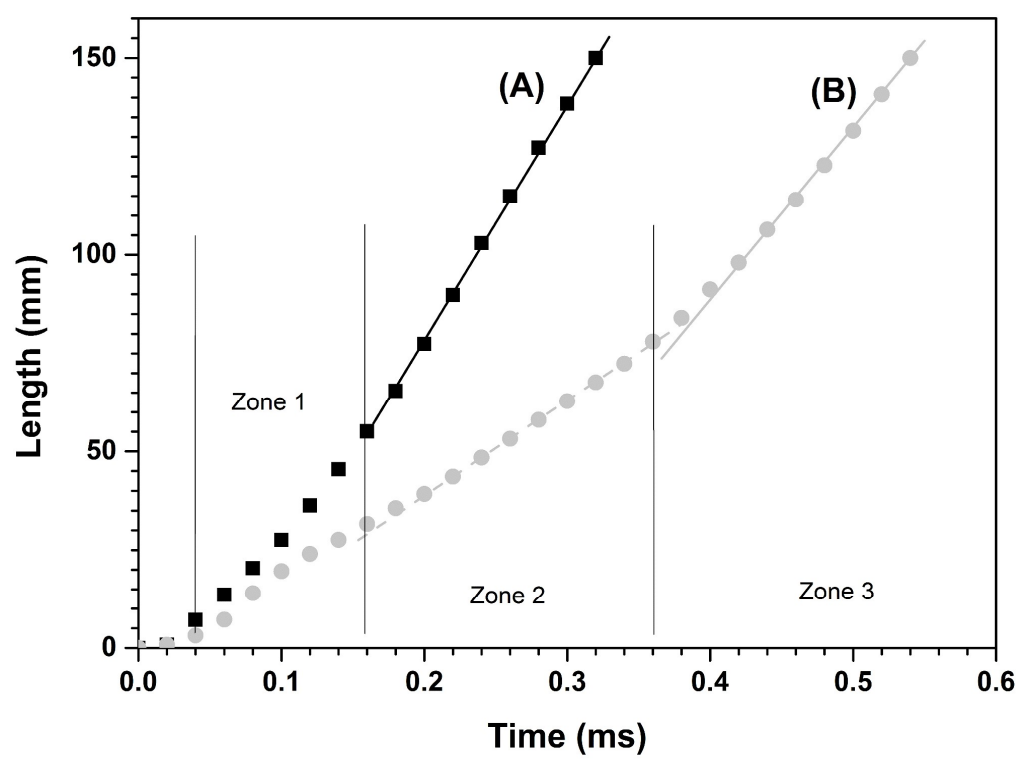

Figure 7: Combustion profiles of the $\mathrm{Al} / \mathrm{WO}_{3}(\mathrm{~A})$ and the $\mathrm{Al} / \mathrm{WO}_{3} / \mathrm{PAni}_{-} \mathrm{LF}$ (B) energetic nanocomposites.

Regarding the $\mathrm{Al} / \mathrm{WO}_{3}$ nanothermite, the calculated combustion velocity is slightly higher than the values reported by Prentice et al. $(310-490 \mathrm{~m} / \mathrm{s})[34]$ for similar energetic 
composites. This difference can be explained by a confinement effect caused by our glass tubes, whereas in the literature, an open-channel testing apparatus was used. Regarding the $\mathrm{Al} / \mathrm{WO}_{3} / \mathrm{PAni}$ LF nanothermite, the increase in the combustion velocity within zone 3 can be interpreted as a pre-heating of the fuel, oxidiser and polymer nanoparticles (unreacted matter) caused by the combustion gaseous products (e.g., $\mathrm{CO}_{\mathrm{x}}, \mathrm{H}_{2} \mathrm{O}, \mathrm{NO}_{\mathrm{x}} .$. ) of the polyaniline component burned inside the third zone. Then, the ignition of the nanocomposites requires less energy to burn; this explains the acceleration of the displacement of the reaction front through the matter. Experiments are currently in progress to refine the understanding of the reaction mechanism that is implied in this polyaniline-based energetic nanocomposite. Finally, note that the combustion velocity of the PAni-doped nanothermites is of the same order of magnitude as that of the conventional binary $\mathrm{Al} / \mathrm{WO}_{3}$ nanothermite. This result represents a truly major scientific breakthrough in the preparation of safer and more reliable reactive nanocomposites because the studies conducted for desensitising energetic nanocomposites and reported in the literature have always led to a drastic loss of reactivity $[16,18]$

\section{Conclusion}

In summary, a tremendous electrostatic desensitisation of a $\mathrm{WO}_{3} / \mathrm{Al}$ nanothermite while maintaining good pyrotechnic properties was achieved using high aspect ratio polyaniline nanofibres. The strong increase in the ESD threshold from $0.14 \mathrm{~mJ}$ to $120 \mathrm{~mJ}$ was obtained with a low mass content of $5 \mathrm{wt} \%$ additive. This value is six times higher than the estimated energy of a spark generated by an ungrounded human body $(20 \mathrm{~mJ})$. Moreover, the mechanical thresholds are higher than the standard values: a complete desensitisation to friction $(>360 \mathrm{~N})$ is obtained while the impact sensitivity remains at a value that is 10 times higher than the international standard value (20 J vs. $2 \mathrm{~J}$ ). Regarding the loose powder 
nanothermite, the combustion velocity is of the same order of magnitude as that of the binary nanothermite when using long PAni nanofibres $(414 \pm 35 \mathrm{~m} / \mathrm{s}$ vs. $620 \pm 5 \mathrm{~m} / \mathrm{s})$. This strong desensitisation (mechanical and electrostatic) coupled with good reactivity outperforms the achievements obtained so far in response to one of the most challenging issues for the development of safer nanothermites for civilian, military and aerospace applications.

\section{Acknowledgements}

The authors gratefully acknowledge the French Defense procurement agency (Direction Générale de l'Armement (DGA)) and the French National Centre for Scientific Research (CNRS) for their financial support.

\section{References}

[1] Regulation 1907/2006, Official Journal of the European Union. 2006. L136/1.

[2] Matyas, R., J. Selesovsky, T. Musil. 2012. Sensitivity to friction for primary explosives, Journal of Hazardous Materials. 213-214:236-241.

[3] Talawar, M.B., A.P. Agrawal, M. Anniyappan, D.S. Wani, M.K. Bansode, G.M. Gore. 2006. Primary explosives: electrostatic discharge initiation, additive effect and its relation to thermal and explosives characteristics. Journal of Hazardous Materials. 137:1074-1078.

[4] Ilyushin, M.A., I.V. Tselinsky, I.V. Shugalei. 2012. Environmentally friendly energetic materials for initiation devices. Central European Journal of Energetic Materials. 9:293-327.

[5] Talawar, M.B., R. Sivabalan, T. Mukundar, H. Muthurajan, A.K. Sikder, B.R. Gandhe, A.S. Rao. 2009. Environmentally compatible next generation green energetic materials (GEMs), Journal of Hazardous Materials. 161:589-607.

[6] Mehrkesh, A., A.T. Karunanthi. 2013. Energetic Ionic Materials: How green are they? A comparative life cycle assessment study. ACS Sustainable Chemistry \& Engineering. 1:448-455.

[7] Steinhauser, G., T.M. Klapötke. 2008. "Green" pyrotechnics: a chemists' challenge. Angewandte Chemie International Edition Engl. 47:3330-3347.

[8] United Nations. 2007. Recommendations on the transport of dangerous goods: Manual of tests and criteria, $4^{\text {th }}$ revised edition, New York and Geneva.

[9] Dixon. 1998. US Patent $n^{\circ} 5,171,159$.

[10] Fischer, S.H., M.C. Grubelich. 1998. Theoretical energy release of thermites, intermetallics, and combustible metals. Proceedings of the $24^{\text {th }}$ International Pyrotechnics Seminar, pp 231-286, July 27-31, Monterey, USA. 
[11] Piercy D.G., Klapotke T.M. 2010. Nanoscale Aluminum - Metal Oxide (Thermite) reactions for application in energetic materials. Central European Journal of Energetic Materials. 7:115-129.

[12] Wang, L., D. Luss, K.S. Martirosyan. 2011. The behavior of nanothermite reaction based on $\mathrm{Bi}_{2} \mathrm{O}_{3} / \mathrm{Al}$. Journal of Applied Physics. 110:074311.

[13] Shende, R., S. Subramanian, S. Hasan, S. Apperson, R. Thiruvengadathan, R. Gangopadyay, S. Gangopadhyay. 2008. Nanoenergetic Composites of CuO Nanorods, Nanowires, and Al-Nanoparticles. Propellants, Explosives, Pyrotechnics. 33:122-130.

[14] Poper, K.H., E.S. Collins, M.L. Pantoya, M.A. Daniels. 2014. Controlling the electrostatic discharge ignition sensitivity of composite energetic materials using carbon nanotube additives. Journal of Electrostatics. $72: 428-432$.

[15] Collins, E.S., B.R. Skelton, M.L. Pantoya, F. Irin, M.J. Green. 2015. Ignition sensitivity and electrical conductivity of an aluminum fluoropolymer reactive material with carbon nanofillers. Combustion and Flame. 162:1417-1421.

[16] Steelman, R., B. Clark, M.L. Pantoya, R.J. Heaps. 2015. Desensitizing nanopowders to electrostatic discharge ignition. Journal of Electrostatics. 76:102-107.

[17] Kappagantula, K., M.L. Pantoya, E.M. Hunt. 2012. Impact ignition of aluminum-teflon based energetic materials impregnated with nano-structured carbon additives. Journal of Applied Physics. 112:024902. [18] Siegert, B., M. Comet, O. Muller, G. Pourroy, D. Spitzer. 2010. Reduced-sensitivity Nanothermites containing manganese oxide filled carbon nanofibers. The Journal of Physical Chemistry. 114:19562-19568.

[19] Foley, T., A. Pacheco, J. Malchi, R. Yetter, K. Higa. 2007. Development of nanothermite composites with variable electrostatic discharge ignition thresholds. Propellants, Explosives, Pyrotechnics. 32:431-434.

[20] Bhadra, S., D. Khastgir, N.K. Singha, J.H. Lee. 2009. Progress in preparation, processing and applications of polyaniline. Progress in Polymer Science. 34:783-810.

[21] Ciric-Marjanovic, G. 2013. Recent advances in polyaniline research: polymerization mechanisms, structural aspects, properties and applications. Synthetic Metals. 177:1-47.

[22] Song, G., J. Han, J. Bo, R. GuO. 2009. Synthesis of polyaniline nanostructures in different lamellar liquids crystals and application to lubrication. Journal of Materials Science. 44:715-720.

[23] Rahy, A., D.J. Yang. 2008. Synthesis of highly conductive polyaniline nanofibers. Materials Letters. 62:4311-4314.

[24] Rahy, A., M. Sakrout, S. Manohar, S.J. Cho, J. Ferraris, D.J. Yang. 2008. Polyaniline nanofibers synthesis by co-use of ammonium peroxydisulfate and sodium hypochlorite. Chemistry Materials. 20:4808-4814.

[25] NATO. 1999. STANAG 4489. In: Standardization Agreement (STANAG) on Explosives, Impact Sensitivity Tests. NATO Standardization Agency: Brussels, Belgium.

[26] NATO. 2002. STANAG 4487. In: Standardization Agreement (STANAG) on Explosives, Friction Sensitivity Tests. NATO Standardization Agency: Brussels, Belgium.

[27] NATO. 2001. STANAG 4490. In: Standardization Agreement (STANAG) on Explosives, Electrostatic Discharge Sensitivity Tests. NATO Standardization Agency: Brussels, Belgium.

[28] Comet, M., F. Schnell, V. Pichot, J. Mory, B. Risse, D. Spitzer. 2014. Boron as Fuel for Ceramic Thermites. Energy \& Fuel. 28:4139-4148. 
[29] Greason, W.D. 2003. Electrostatic discharge characteristics for the human body and circuit packs. Journal of Electrostatics. 53:285-300.

[30] Hardt, A.P. 1988. Shock initiation of thermite, Proceedings of the $13^{\text {th }}$ International. Pyrotechnics Seminar. pp 425-438, July 11-15, Grand Junction, USA.

[31] J.J. Davis, D.L. Woody. 1995. Reactions in neat porous metal/metal and metal/metal oxide mixtures under shear induced plastic flow conditions, pp 661-668, Amsterdam, Elsevier.

[32] Gibot, P., M. Comet, A. Eichhorn, F. Schnell, O. Muller, F. Ciszek, Y. Boehrer, D. Spitzer. 2011. Highly insensitive/reactive thermite prepared from $\mathrm{Cr}_{2} \mathrm{O}_{3}$ nanoparticles. Propellants, Explosives, Pyrotechnics. 36:8087.

[33] Gibot, P., M. Comet, L. Vidal, F. Moitrier, F. Lacroix, Y. Suma, F. Schnell, D. Spitzer. 2011. Synthesis of $\mathrm{WO}_{3}$ nanoparticles for superthermites by the template method from silica spheres. Solid State Science. 13:908914.

[34] Prentice, D., M.L. Pantoya, A.E. Gash. 2006. Combustion wave speeds of sol-gel synthesized tungsten trioxide and nano-aluminium: the effect of impurities on flame propagation. Energy \& Fuel. 20:2370-2376. 